most upper-tropospheric heating in the tropics is advected out of the region, $\mathrm{CO}_{2}$ warming in the atmosphere should have much less of an effect on limiting the SST than does direct solar heating, although the relationship between atmospheric warming and SST is a subject of controversy. In addition, atmos- pheric warming by $\mathrm{CO}_{2}$ may alter the vertical temperature profile, leading to changes in the atmospheric stability.

Andrew J. Heymsfield and Larry $M$. Miloshevich are at the National Center for Atmospheric Research, Boulder, Colorado 80307, USA.

\title{
Shedding light on alignment
}

\section{Cliff Jones and Sally Day}

ORGANIC materials have enormous potential for use in electro-optic and opto-optic devices. Nowhere is this more evident than with liquid crystals, which are already familiar in the displays of digital watches and laptop computers. These devices are electro-optic: applying an electric field reorientates the liquid crystal molecules, thereby altering the device's optical properties. New effects and applications are being sought, and one particularly exciting possibility is that of controlling the molecular orientation using incident light. Such an effect is described by Gibbons et al. on page 49 of this issue ${ }^{1}$, who show how to manipulate the orientation of a nematic liquid crystal by irradiating the surface alignment layer with polarized light.

Vital to most liquid crystal devices are surface alignment agents which define the molecular orientation of the device in the unswitched state. Numerous surfactants are available, but the usual commercial technique is to coat the inner surfaces of the glass cell containing the liquid crystals with a polymer such as polyimide. This causes the rod-like liquid crystal molecules to lie parallel to the surface. Rubbing the surface with a nylon cloth then imparts a preferred alignment for the liquid crystal molecules once the cell is filled. For example, in the common twisted-nematic device, the surfaces are rubbed at $90^{\circ}$ to each other, so that the liquid crystal molecules twist through this angle from one surface to the other. Light is transmitted through the liquid crystals when viewed between crossed polarizers, and the cell appears light. Applying a small voltage (typically about a volt) reorientates the molecules, removing the twisted structure so that the device is dark. In this manner the twisted-nematic cell acts as a simple electro-optic shutter.

Gibbons et al. use a novel alignment layer that is a mixture of polyimide and an azodye. Anisotropic dyes of this type are often used in liquid crystal applications because they align parallel to the liquid crystal molecules, providing anisotropic absorption of incident light and allowing displays incorporating a single polarizer. Gibbons et al. constructed a cell in which one surface is coated with polyimide and the other with the dye-doped polyimide, and the plates arranged with rubbing directions parallel. The liquid crystal is then uniform and behaves optically as a birefringent slab (so that, for

example, it would appear dark between crossed polarizers). Incident light from an argon-ion laser, polarized parallel to the initial direction of alignment, causes the liquid crystal molecules at the dye-doped polyimide surface to reorientate through $90^{\circ}$, forming a twisted-nematic structure which transmits light between crossed polarizers.

Clearly, this effect is due to the presence of the dye in the alignment layer. Similar effects have been observed in the bulk of azo-dye-doped monomeric ${ }^{2}$ and side-chain polymeric $^{3}$ liquid crystals. Anderle et al. ${ }^{4}$ suggest a mechanism for the photoinduced reorientation of the liquid crystal based on trans-cis isomerization of the azo-dye, changing the molecules' shape (see figure). Illuminating the dye with light of wavelength close to its absorption maximum induces the trans-cis reaction, with highest reaction probability for molecules parallel to the plane of polarization of the incident light because of the anisotropy of the dye's absorb-

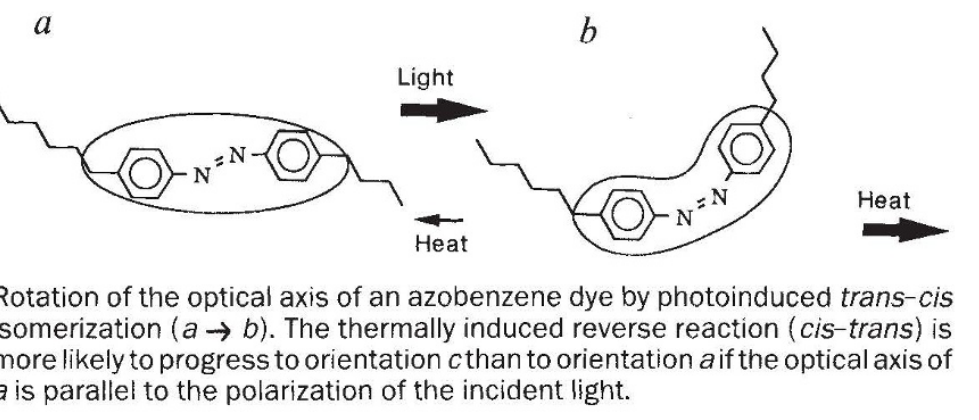

ponse of the reorientation process.

Photoinduced isomerism may also lead to the surface alignment effects described by Gibbons et al. Two mechanisms have been proposed for the alignment of liquid crystals on rubbed polymers. One involves a longrange interaction due to surface microgrooves caused by the rubbing. The elastic energy of the liquid crystal is then minimized if the molecules lie along the grooves. Alternatively, the alignment mechanism may be a short range interaction between the liquid crystal molecules and the polymer surfactant. It is possible to distinguish between these mechanisms through measurement of the optical second-harmonic generation (SHG, a frequency-doubling effect) of the noncentrosymmetric monolayer at a surface $^{5}$. The liquid-crystal/rubbed-polymer interface has been studied using this powerful technique by Chen et al. ${ }^{6}$, who show, from the symmetry and polarization dependence of the SHG signal, a preferential, polar orientation of the surface layer of crystal along the rubbing direction of polyimide. Other alignment layers do not show such short-range, polar interaction and it is presumed that the alignment mechanism is due solely to the longer range elastic forces in the liquid crystal bulk. Although a dyed polyimide surfactant has not been studied by this method, the results of Gibbons et al. indicate that the liquid crystal molecules interact even more strongly with the dye than the polyimide.

Interesting new uses of liquid crystals may be emerging that take advantage of the fine,

ance. The thermally excited reverse reaction, cis-trans, may occur by either of two routes but the final state is most likely to be perpendicular to the polarization of the incident light because of the continued excitation of the molecules in that direction.

A liquid crystal mixed with the dye will also become reoriented under these circumstances, owing to the molecular interaction between the dye and the liquid crystal, and therefore the optical axis of the sample will rotate to be perpendicular to the incident polarized light. Eich and Wendorff ${ }^{3}$ have begun to demonstrate the considerable potential for these effects in liquid crystal polymers as a medium of eraseable optical storage, producing diffraction gratings and holograms. The possibility of real-time holography for display purposes in monomeric liquid crystals $^{2}$ is however limited by the slow re- adaptive control of the orientation of liquid crystals using light instead of the conventional fixed electrode structures. But the mechanisms involved must be understood more fully in order to optimize the efficiency, response times and optical characteristics needed for practical devices.

Cliff Jones and Sally Day are at the Electronics Division of the Defence Research Agency, RSRE, St Andrews Road, Malvern, Worcestershire WR14 3PS, UK.

\footnotetext{
Gibbons, W. M., Shannon, P. J., Sun, S. \& Swetlin, B. J. Nature 351, 49-50 (1991).

2. Anderle, $\mathrm{K}$. et al. Proc. 20th Freiburger Arbeitstagung Flussigkristalle, P22 (Fraunhofer Inst., Freiburg, 1991)

3. Eich, M. \& Wendorff, J. H. J. opt. Soc. Am. B7, 1428-1436 (1990)

4. Anderle, K. et al. Liquid Crystals, 9, 691-699 (1991).

5. Shen, Y. R. Nature 337, 519-525 (1989).

6. Chen, W., Feller, M. B. \& Shen, Y. R. Phys. Rev. Lett 63 2665-2668 (1989)
} 